\title{
Startup Transient Response Analysis of an Energy Storage Flywheel System
}

\author{
Wang Hongchang ${ }^{a}$ * , Du Zhouming ${ }^{b}$ \\ School of Mechanical Engineering, Jiangsu University of Technology, Changzhou,213001, China \\ *ahnwanghc@163.com, duzhouming@jsut.edu.cn
}

\begin{abstract}
Keywords: energy storage flywheel; transient responses; transfer matrix method; Wilson- $\theta$ formulation integration method
\end{abstract}

\begin{abstract}
In order to analyze the startup transient response of a flywheel system, its shaft system is studied in this paper: The transient oil forces of a squeeze film damper (SFD) in fixed frame are firstly derived. By putting the Wilson- $\theta$ formulation integration method which having damp of algorithm into the motion equations of a rigid disc's barycenter, the transient transfer matrix of a station made of a disc and a shaft is also built which can improve algorithm stability. The transient-state response equations of the flywheel shaft system are built and solved by putting Taylor series and numerical iteration into the transfer matrix method, and the startup transient-state response curves of rotor with different constant angular acceleration are finally obtained. The calculation results show that the theoretical method introduced in this paper can be used to analyze the performance of quick charge of a flywheel shaft system.
\end{abstract}

\section{Introduction}

Comparing with other storing units, the energy storage flywheel (ESF) is very attractive in recent years on account of its high efficiency, short recharge time, universal localization, environment-friendly, high power density [1-3]. The existing literatures on the analysis of it were concentrated on application, control, mechanism innovation and steady unbalance responses [4-7], however, research on transient dynamics analysis was rarely involved. In this paper, the hybrid bearings consisting of a permanent magnetic bearing (PMB) and two angular contact ceramic ball bearings (ACBB) were used as the support for the flywheel system, and we carried on the theoretical analysis and study aiming at the performance of quick charge, namely startup transient dynamic analysis.

The transient response of rotor-bearing system can be classified as seeking initial value problem in mathematics. In order to facilitate the analysis, the dynamic equations are often built in stationary reference frame and there are several methods often utilized such as, using third party software (for instance ANSYS) [8], introducing numerical integration method into the Lagrange equation [9], and introducing numerical integration into the finite element method [10]. The three methods all have some disadvantages, the calculation accuracy solved by ANSYS software is low, the Lagrange equation method is too complicated and the finite element method has low computational efficiency. The Prohl transfer matrix method (TMT) has advantages of simple programming, quick computing speed, so the transient-state response equation of the flywheel system is built and solved by means of putting the Wilson- $\theta$ formulation integration method into the Prohl transfer matrix method: firstly, the oil film force of the squeeze film damper (SFD) and $9 \times 9$ order transient transfer matrix of a station made of a disc and a shaft are built in stationary reference frame. Secondly, the transient-state equation of the flywheel system crossing the critical speed with constant acceleration is formed by Phrol TMT. Thirdly, the transient-state equation of the ESF is solved by the transient TMT, Taylor series and numerical iteration. Finally, the theoretical analysis of the performance of quick charge is studied. 


\section{The Transient-state Dynamic Equation of the Flywheel System}

Oil Film Forces in Stationary Reference Frame. In present literatures, the equations of SFD's transient oil film forces are all built in rotating frame. In order to build the transient-state equation of the flywheel system, oil film forces of SFD in stationary reference frame should be firstly derived.

According to relationship of oil film forces in stationary and rotating frame, we can obtain

$$
F=F_{\mathrm{x}}+\mathrm{i} F_{\mathrm{y}}=-\left(F_{\mathrm{r}}+\mathrm{i} F_{\mathrm{t}}\right) e^{\mathrm{i} \psi}
$$

Where the mathematical expressions of $F_{\mathrm{t}} 、 F_{\mathrm{r}}$ can be referred in Reference [9].

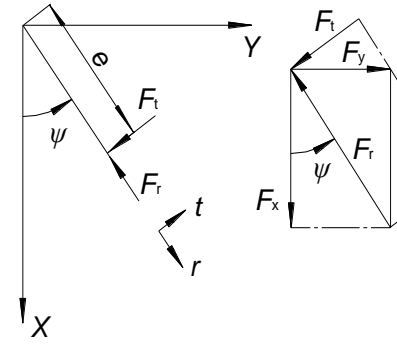

Figure 1. The coordinate transformation of oil film forces

Due to the relationship between stationary and rotating frame, we can also obtain

$$
\begin{aligned}
& x+\mathrm{i} y=e e^{\mathrm{i} \psi}, \quad x=e \cos \psi, \quad y=e \sin \psi \\
& e=\left(x^{2}+y^{2}\right)^{\frac{1}{2}}, \quad \dot{e}=\frac{1}{e}(x \dot{x}+y \dot{y})
\end{aligned}
$$

Putting Eq. 2 and Eq.3 into Eq. 1 yields the equations of transient oil film forces in stationary reference frame.

$$
F_{\mathrm{x}}=f(x, y, \dot{x}, \dot{y}), \quad F_{\mathrm{y}}=f(x, y, \dot{x}, \dot{y})
$$

The Transient Dynamic Equation of a Rigid Disc. The transient response of a rotor-bearing system is no longer simple harmonic motion and the solution of the transient equation cannot be expressed as $\{x\}=\{A\} \mathrm{e}^{\lambda t}$, so the transfer matrix introduced by the References $[11,12]$ is no longer suitable. Therefore, the transient transfer matrix of a station made of a disc and a shaft is firstly built in stationary reference frame considering the effects of oil film forces of the SFD in this paper.

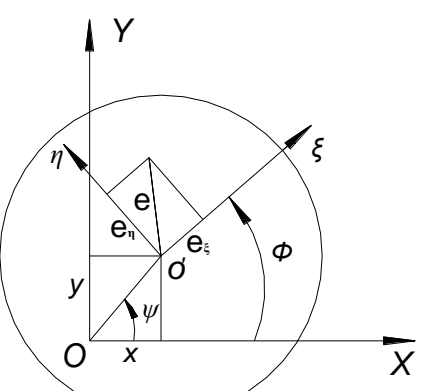

Figure 2. The coordinator frames of a rigid disc

The geometric relationship between barycenter and section center of a rigid disc is shown in Fig.2. We can see inertial frame oxyz with origin in the rest position of the supported bearings and rotating frame $o^{\prime} \xi \eta$ with origin in $o^{\prime} . c$ and $o^{\prime}$ respectively represent the barycenter and section center of the disc [11]. From Fig.2 we can obtain

$$
\begin{aligned}
& x_{\mathrm{c}}=x+e_{\xi} \cos \omega t-e_{\eta} \sin \omega t \\
& y_{\mathrm{c}}=y+e_{\xi} \sin \omega t+e_{\eta} \cos \omega t
\end{aligned}
$$

The speed and acceleration equations of the rigid disc can be obtained by the first derivative and second derivative 


$$
\begin{aligned}
& \dot{x}_{\mathrm{c}}=\dot{x}-e_{\xi} \omega \sin \omega t-e_{\eta} \omega \cos \omega t \\
& \dot{y}_{\mathrm{c}}=\dot{y}+e_{\xi} \omega \cos \omega t-e_{\eta} \omega \sin \omega t \\
& \ddot{x}_{\mathrm{c}}=\ddot{x}-\left(e_{\xi} \omega^{2}+e_{\eta} \varepsilon\right) \cos \omega t+\left(e_{\eta} \omega^{2}-e_{\xi} \varepsilon\right) \sin \omega t \\
& \ddot{y}_{\mathrm{c}}=\ddot{y}+\left(e_{\xi} \varepsilon-e_{\eta} \omega^{2}\right) \cos \omega t-\left(e_{\xi} \omega^{2}+e_{\eta} \varepsilon\right) \sin \omega t
\end{aligned}
$$
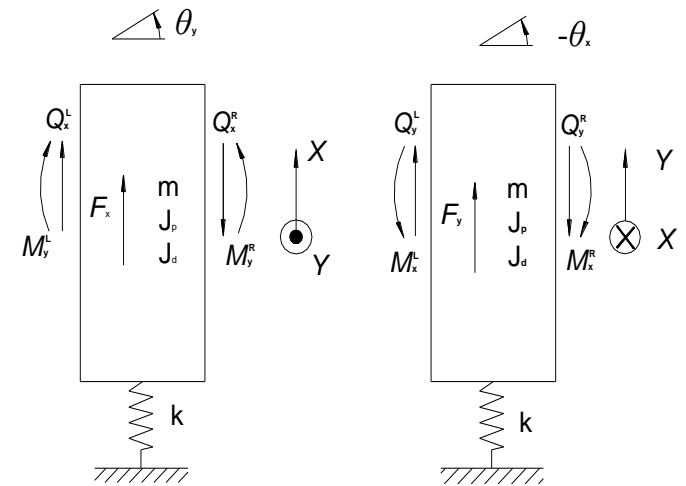

Figure 3. The schematic view of a rigid disc in $o x z$ and $o y z$ planes

Fig. 3 shows a schematic view of the rigid disc in oxz and oyz planes. The motion equations built by D'Alembert's principle are as follows [10]

$$
\begin{aligned}
& m \ddot{x}_{c}=-k x+Q_{x}^{L}-Q_{x}^{R}+F_{x} \\
& m \ddot{y}_{c}=-k y+Q_{y}^{L}-Q_{y}^{R}+F_{y} \\
& J_{d} \ddot{\theta}_{y}-J_{p} \omega \dot{\theta}_{x}-J_{p} \varepsilon \theta_{x}=M_{y}^{R}-M_{y}^{L} \\
& J_{d} \ddot{\theta}_{x}+J_{p} \omega \dot{\theta}_{y}+J_{p} \varepsilon \theta_{y}=M_{x}^{R}-M_{x}^{L}
\end{aligned}
$$

Wilson- $\boldsymbol{\theta}$ Formulation Integration Method. In order to avoid the algorithm losing its stability as the responses having high frequency components, Wilson- $\theta$ implicit formulation integration method which having damp of algorithm is used. It assumes the acceleration varies linearly at the time interval from $t$ to $t+\theta \Delta t$, and the generalized velocity and acceleration at time $t+\theta \Delta t$ can be described as

$$
\begin{aligned}
& \dot{q}_{\mathrm{t}+\theta \Delta \mathrm{t}}=\frac{3}{\theta \Delta t}\left(q_{\mathrm{t}+\theta \Delta \mathrm{t}}-q_{\mathrm{t}}\right)-2 \dot{q}_{\mathrm{t}}-\frac{1}{2} \theta \Delta t \ddot{q}_{\mathrm{t}} \\
& \ddot{q}_{\mathrm{t}+\Delta \Delta \mathrm{t}}=\frac{6}{(\theta \Delta t)^{2}}\left(q_{\mathrm{t}+\theta \Delta \mathrm{t}}-q_{\mathrm{t}}\right)-\frac{6}{\theta \Delta t} \dot{q}_{\mathrm{t}}-2 \ddot{q}_{\mathrm{t}}
\end{aligned}
$$

The generalized velocity and acceleration at time $t+\Delta t$ are as follows

$$
\begin{aligned}
& \ddot{q}_{\mathrm{t}+\Delta \mathrm{t}}=\frac{6}{\theta^{3} \Delta t^{2}}\left(q_{\mathrm{t}+\Delta \Delta \mathrm{t}}-q_{\mathrm{t}}\right)-\frac{6}{\theta^{2} \Delta t} \dot{q}_{\mathrm{t}}+\left(1-\frac{3}{\theta}\right) \ddot{q}_{\mathrm{t}} \\
& \dot{q}_{\mathrm{t}+\Delta \mathrm{t}}=\dot{q}_{\mathrm{t}}+\frac{\Delta t}{2}\left(\ddot{q}_{\mathrm{t}+\Delta \mathrm{t}}+\ddot{q}_{\mathrm{t}}\right) \\
& q_{\mathrm{t}+\Delta \mathrm{t}}=q_{\mathrm{t}}+\dot{q}_{\mathrm{t}} \Delta t+\frac{\Delta t^{2}}{6}\left(\ddot{q}_{\mathrm{t}+\Delta \mathrm{t}}+2 \ddot{q}_{\mathrm{t}}\right)
\end{aligned}
$$

When $\theta>1.3$, the algorithm is unconditional stability. In this paper, we take the $\theta$ value is 1.4 .

The Transient Transfer Matrix of a Station Made of a Disc and a Shaft. Putting Eq.4, Eq.6 and Eq.9 into Eq. 8 and introducing state vector $\mathrm{Z}$ yields

$$
\mathrm{Z}_{\mathrm{t}+\Delta \Delta t}^{\mathrm{R}}=[D] Z_{t+\Delta \Delta t}^{\mathrm{L}}
$$

Where, $\mathrm{Z}=\left[\begin{array}{lllllllll}x & \theta_{\mathrm{x}} & M_{\mathrm{x}} & Q_{\mathrm{x}} & y & \theta_{\mathrm{y}} & M_{\mathrm{y}} & Q_{\mathrm{y}} & 1\end{array}\right]^{\mathrm{T}}$, [D] is the transient transfer matrix of a rigid disc.

The transient transfer matrix of a massless elastic shaft with equal section is identical to the steady transfer matrix [9]. Suppose the transfer matrix of it is [B], the transient transfer matrix of a station made of a rigid disc and an elastic shaft can be described as follows 


$$
[T]=[B][D]=\left[\begin{array}{ccc}
T_{11} & T_{12} & T_{13} \\
T_{21} & T_{22} & T_{23} \\
0 & 0 & 1
\end{array}\right]
$$

Where, $A_{1}=\frac{6 \mathrm{~J}_{\mathrm{d}}}{(\theta \Delta t)^{2}}, A_{2}=-\frac{6 \mathrm{~m}}{(\theta \Delta t)^{2}}-\mathrm{k}$

$$
\begin{aligned}
& C_{1}\left(\mathrm{C}_{3}\right)=-\frac{6 \mathrm{~J}_{\mathrm{d}}}{(\theta \Delta t)^{2}} \theta_{\mathrm{x}(\mathrm{t})}\left(\theta_{\mathrm{y}(\mathrm{t})}\right)-\frac{6 \mathrm{~J}_{d}}{\theta \Delta t} \dot{\theta}_{\mathrm{x}(\mathrm{t})}\left(\dot{\theta}_{\mathrm{y}(\mathrm{t})}\right)-2 \mathrm{~J}_{\mathrm{d}} \ddot{\theta}_{\mathrm{x}(\mathrm{t})}\left(\ddot{\theta}_{\mathrm{y}(\mathrm{t})}\right)-\frac{3 \mathrm{~J}_{\mathrm{p}} \omega}{\theta \Delta t} \theta_{\mathrm{y}(\mathrm{t})}\left(-\theta_{\mathrm{x}(\mathrm{t})}\right)-2 \mathrm{~J}_{\mathrm{p}} \omega \dot{\theta}_{\mathrm{y}(\mathrm{t})}\left(-\dot{\theta}_{\mathrm{x}(\mathrm{t})}\right)-\frac{1}{2} \mathrm{~J}_{\mathrm{p}} \omega \theta \Delta t \ddot{\theta}_{\mathrm{y}(\mathrm{t})}\left(-\ddot{\theta}_{\mathrm{x}(\mathrm{t})}\right) \\
& C_{2}\left(\mathrm{C}_{4}\right)=\frac{6 \mathrm{~m}}{(\theta \Delta t)^{2}} x_{\mathrm{t}}\left(y_{\mathrm{t}}\right)+\frac{6 \mathrm{~m}}{\theta \Delta t} \dot{x}_{\mathrm{t}}\left(\dot{\mathrm{y}}_{\mathrm{t}}\right)+2 \mathrm{~m} \ddot{x}_{\mathrm{t}}\left(\ddot{\mathrm{y}}_{\mathrm{t}}\right)+F_{\mathrm{x}}\left(F_{\mathrm{y}}\right)+\mathrm{m} e_{\xi} \varepsilon \sin \omega t(-\cos \omega t)+\mathrm{m} e_{\xi} \omega^{2} \cos \omega t(\sin \omega t) \\
& +\mathrm{m} e_{\eta} \varepsilon \cos \omega t(\sin \omega t)-\mathrm{m} e_{\eta} \omega^{2} \sin \omega t(-\cos \omega t)
\end{aligned}
$$

Where, $\left[T_{11}\right]=\left[T_{22}\right]=\left[\begin{array}{cccc}1+\frac{l^{3}(1-v)}{6 \mathrm{EI}} A_{2} & l+\frac{l^{2}}{2 \mathrm{EI}} A_{1} & \frac{l^{2}}{2 \mathrm{EI}} & \frac{l^{3}(1-v)}{6 \mathrm{EI}} \\ \frac{l^{2}}{2 \mathrm{EI}} A_{2} & 1+\frac{l}{\mathrm{EI}} A_{1} & \frac{l}{\mathrm{EI}} & \frac{l^{2}}{2 \mathrm{EI}} \\ l A_{2} & A_{1} & 1 & l \\ A_{2} & 0 & 0 & 1\end{array}\right], \quad\left[T_{12}\right]=-\left[T_{21}\right]=\left[\begin{array}{cccc}0 & \frac{l^{2}}{2 \mathrm{EI}}\left(\frac{3 \mathrm{~J}_{\mathrm{p}} \omega}{\theta \Delta t}+\mathrm{J}_{\mathrm{p}} \varepsilon\right) & 0 & 0 \\ 0 & \frac{l}{\mathrm{EI}}\left(\frac{3 \mathrm{~J}_{\mathrm{p}} \omega}{\theta \Delta t}+\mathrm{J}_{\mathrm{p}} \varepsilon\right) & 0 & 0 \\ 0 & B_{1} & 0 & 0 \\ 0 & 0 & 0 & 0\end{array}\right]$

$$
\left[T_{13}\right]=\left[\begin{array}{c}
\frac{l^{2}}{2 \mathrm{EI}} C_{1}+\frac{l^{3}(1-v)}{6 \mathrm{EI}} C_{2} \\
\frac{l}{\mathrm{EI}} C_{1}+\frac{l^{2}}{2 \mathrm{EI}} C_{2} \\
C_{1}+l C_{2} \\
C_{2}
\end{array}\right],\left[T_{23}\right]=\left[\begin{array}{c}
\frac{l^{2}}{2 \mathrm{EI}} C_{3}+\frac{l^{3}(1-v)}{6 \mathrm{EI}} C_{4} \\
\frac{l}{\mathrm{EI}} C_{3}+\frac{l^{2}}{2 \mathrm{EI}} C_{4} \\
C_{3}+l C_{4} \\
C_{4}
\end{array}\right]
$$

Solution of the Transient Dynamic Equation . Because oil film forces are the complex nonlinear function of displacement and velocity of the SFD's journal determined by the transient responses, it is hard to solve dynamic equation when they are unknown. According to this situation, the transient dynamic equation is solved by introducing Taylor series and numerical iteration into the transient TMT.

1. Set the scope of time $T$ and step length $\Delta t$.

2. Determine starting values of the displacement, velocity and acceleration of each element node at time $t$ or $t_{0}$.

3. Using Taylor series to estimate the displacement and velocity of the element node supported by SFD: $\{e\}_{\mathrm{t}+\theta \Delta \mathrm{t}}=\{e\}_{\mathrm{t}}+\theta \Delta t\{\dot{e}\}_{\mathrm{t}} 、\{\dot{e}\}_{\mathrm{t}+\theta \Delta \mathrm{t}}=\{\dot{e}\}_{\mathrm{t}}+\theta \Delta t\{\ddot{e}\}_{\mathrm{t}}$, then putting them into Eq.4 solves the oil film forces at time $t+\theta \Delta t$.

4. Use the TMT to solve the displacement at time $t+\theta \Delta t$, and use Eq.9 to solve the velocity at time $t+\theta \Delta t$.

5. Compare the values at the same node and time solved by setp-3 and step-4, If the two values are in close agreement, the values solved by TMT are accepted. If the results do not agree to a specified accuracy, we choose the mathematical mean value of them as new starting values and repeat the steps from 3 to 4 again, until we get the required accuracy.

6. Use Wilson- $\theta$ method to solve the displacement, velocity and acceleration of each element node at time $t+\Delta t$, and then use them as the starting values of the next step, until the transient responses are all obtained.

\section{Numerical Results and Discussion}

The Flywheel System. The physical structure of the ESF is shown in Fig.4 [15]. Two ACBBs are respectively used as the lower and upper support. A PMB above the rotor top surface is also used as the upper support, which provides axial unloading for the lower ACBB, being capable of low 
frictional resistance and long life for the lower ACBB. A pendulum SFD is employed in the flywheel system to suppress the lateral vibration. The rotor constructed of solid steel with a mass of $110 \mathrm{~kg}$ is used, its designed maximum tangential velocity is $330 \mathrm{~m} / \mathrm{s}$ and its operating speed range is 8000-23,000 rpm. Analyze the fast charge performance of it to determine whether it is safe when it crosses the critical speed to arrive the operating speed with a constant acceleration of $20 \pi \mathrm{rad} / \mathrm{s} 2$, namely the rotor arrives $23000 \mathrm{rpm}$ in 10 seconds.

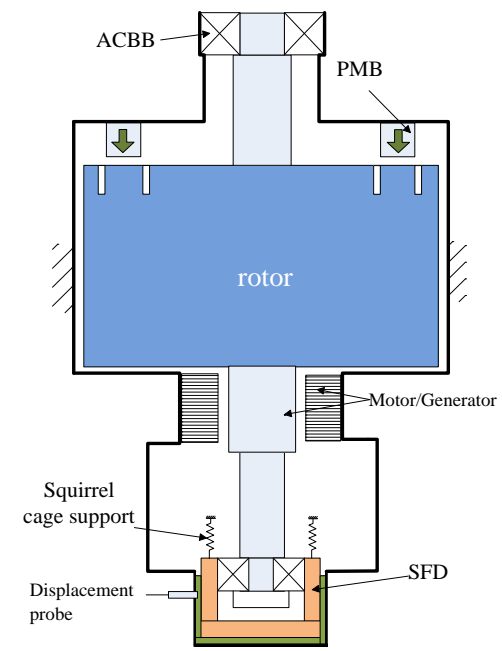

Figure 4. The structure of the flywheel system

Dynamic Model. In the dynamic model of the flywheel system, the following simplifications are made:

1) The stiffness of the lower ACBB in the SFD is much higher than that of the squirrel cage support and the oil film. Therefore, ACBB is fixed to the lower end of the shaft.

2) Comparing with the lateral vibrations, the axial vibrations of the nodes on the central axis are very small and neglected.

3) The stiffness of the upper ACBB with little changes in the operating speed range is considered as constant value.

Table 1. the parameters and values of the flywheel system [15]

\begin{tabular}{c|c|c|c|c}
\hline & $\begin{array}{c}\text { Diameter } \\
\text { (shaft)[m] }\end{array}$ & $\begin{array}{c}\text { Length } \\
\text { (shaft)[m] }\end{array}$ & $\begin{array}{c}\text { Diameter } \\
\text { (disc) }[\mathrm{m}]\end{array}$ & $\begin{array}{c}\text { Thickness } \\
\text { (disc) }[\mathrm{m}]\end{array}$ \\
\hline 1 & 0.02 & 0.01 & 0 & 0 \\
2 & 0.018 & 0.01 & 0 & 0 \\
3 & 0.019 & 0.042 & 0 & 0 \\
4 & 0.1 & 0.05 & 0.3 & 0.05 \\
5 & 0.1 & 0.05 & 0.3 & 0.05 \\
6 & 0.1 & 0.05 & 0.3 & 0.05 \\
7 & 0.1 & 0.05 & 0.3 & 0.05 \\
8 & 0.017 & 0.0115 & 0 & 0 \\
9 & 0.02 & 0.034 & 0 & 0 \\
10 & 0.02 & 0.034 & 0 & 0 \\
11 & 0.02 & 0.034 & 0 & 0 \\
12 & 0.019 & 0.016 & 0 & 0 \\
13 & 0.017 & 0.022 & 0 & 0 \\
14 & 0.017 & 0.022 & 0 & 0 \\
15 & 0.02 & 0.01 & 0 & 0 \\
\hline
\end{tabular}

According to the physical structure of the flywheel system, the rotor-bearing system was divided into several sections and each section is modeled as a massless elastic shaft with lumped masses at the end. Fig. 5 shows the dynamic model and Table 1 shows the parameters and values of the flywheel system. 


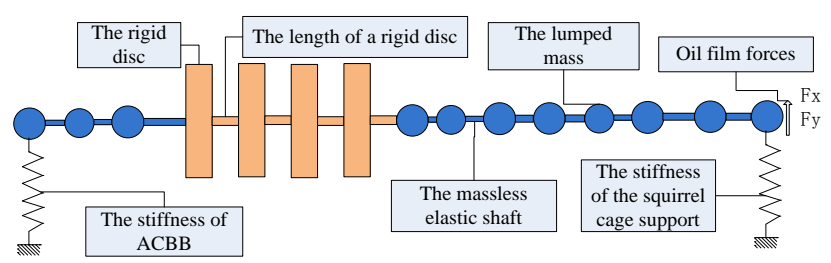

Figure 5. The dynamic model of the flywheel system

The calculation results and discussion. The transient response equation of the flywheel system is solved, and the amplitude-frequency response curves of the rotor top are drawn in Fig.6.

As shown in Fig.6, the first critical speed values increase and the corresponding peak amplitudes reduce with increasing angular acceleration. Compared with the steady-state unbalance response value, the first critical speed values increase by $0.25 \%, 0.95 \%, 2.40 \%, 4.31 \%$ and $7.01 \%$, while the corresponding amplitudes of the rotor top decrease by $42.59 \%, 51.67 \%, 63.52 \%, 71.48 \%$ and $78.33 \%$ when the angular acceleration values are respectively $\pi, 2 \pi, 5 \pi^{2}, 10 \pi$ and $20 \pi \mathrm{rad} \cdot \mathrm{s}^{-2}$. The peak amplitude of rotor is gradually reduced with increasing angular acceleration, so rotor crossing the critical speed with a large angular acceleration is often used in practical engineering to improve stability of the rotor-bearing system.

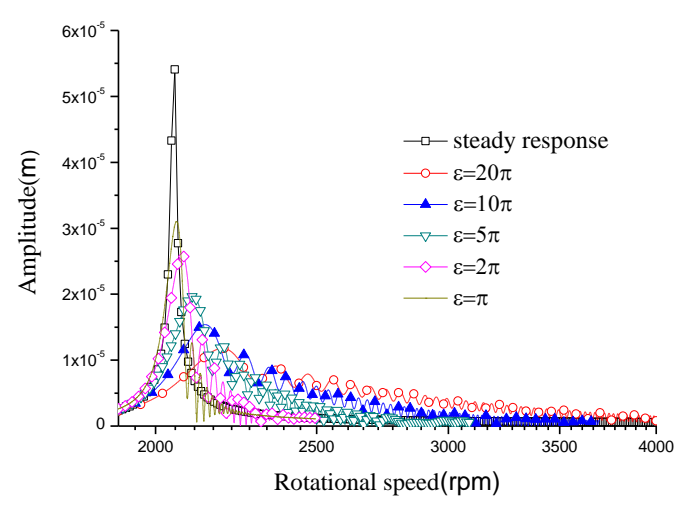

Figure 6. The transient response curves of the rotor top

The oscillations which are actually the result of a spiral motion of the system taking place about the self-centered position become stronger with increasing acceleration. The self-centered conditions are reached after some oscillations are damped out [16].

The calculation results prove the flywheel system is safe when it accelerates from standstill to the operating speed with a big constant acceleration, In another word, the rotor-bearing system accelerates from standstill to the operating speed $(23000 \mathrm{rpm})$ in 8.8 seconds with acceleration value of $20 \pi \mathrm{rad} \cdot \mathrm{s}^{-2}$, which meets the design requirement of fast charge.

\section{Conclusions}

1) According to relationship of oil film forces of SFD in stationary and rotating frame, the calculation equations of oil film forces in stationary frame are derived.

2) Considering the effect of SDF as external forces, the dynamic equation of a rigid disc is built by D'Alembert's principle. The $9 \times 9$ order transient transfer matrix [T] of a station made of a rigid disc and a massless elastic shaft is also built by Wilson- $\theta$ formulation integration method, which can improve the computational stability.

3) The calculation results show that the theoretical method introduced in this paper can be used to analyze the performance of quick charge of a flywheel shaft system.

\section{References}

[1] R.Sebastian, R.Pena Alzola, Flywheel energy storage systems: Review and simulation for an isolated wind power system, Renewable and Sustainable Energy Reviews. 16(2012)6803-6813. 
[2] Ghada Boukettaya, Lotfi Krichen, A dynamic power management strategy of a grid connected hybrid generation system using wind photovoltaic and Flywheel Energy Storage System in residential applications, Energy. 71(2014)148-159.

[3] ZHANG Song, ZHANG Wei-yu, Application status of flywheel energy storage system,Chinese Journal of Power Sources. 36(2012)435-439..

[4] Pan Zhao, Yiping Dai, Jiangfeng Wang, Design and thermodynamic analysis of a hybrid energy storage system based on A-CAES (adiabatic compressed air energy storage) and FESS(flywheel energy storAage system) for wind power application, Energy.70 (2014) 674-684.

[5] R. Sebastián, R. Peña-Alzola, Control and simulation of a flywheel energy storage for a wind diesel power system, Electrical Power and Energy Systems. 64 (2015) 1049-1056.

[6] Jiang Shuyun, Ju Lihua, Study on elctromechanical coupling nonlinear vibration of flywheel energy storage system, Science in China. 49(2006) 61-77.

[7] Hongchang Wang, Shuyun Jiang, Zupei Shen, The dynamic analysis of an energy storage flywheel system with hybrid bearing support, Journal of Vibration and Acoustics. ASME, 131(2009) 051006-1-9.

[8] Zengli Wang, Xiaoling Yu, Feilong Liu, et al, Dynamic analyses for the rotor-journal bearing system of a variable speed rotary compressor, International Journal of Refrigeration. 36 (2013): 1938-1950.

[9] Qinkai Han, Fulei Chu, Dynamic behaviors of a geared rotor system under time-periodic base angular motions, Mechanism and Machine Theory. 78 (2014) 1-14.

[10] An Sung Lee, Byung Ok Kim, Yeong-Chun Kim, A finite element transient response analysis method of a rotor-bearing system to base shock excitations using the state-space Newmark scheme and comparisons with experiments, Journal of Sound and Vibration. 2006 (297) 59-615.

[11] Wen Bang-chun, Gu Jia-liu, Xia Song-bo, et al, Advanced Rotor Dynamics-Theory, Technology and Application, China Machine Press, Beijing, 2000.

[12] Giancarlo Genta. Dynamics of rotating systems, Springer-Verlag, New York, 2004.

[13]P B Bornemann, U Galvanetto, M A Crisfield, Some remarks on the numerical time integration of non-linear dynamical systems, Journal of Sound and Vibration. 252(2002) 935-944.

[14]Z Gu, X Zhi, G Meng, et al, Transient response analysis of large-scale rotor-bearing system with strong non-lonear elements by a transfer matrix-Newmark formulation integration method, Journal of Sound and Vibration. 259(2003) 559-570.

[15] WANG Hongchang. Rotor dynamic analysis and experimental study of flywheel energy storage systems, Southeast University, Nanjing, 2012.

[16]G Genta, C Delprete, Acceleration through critical speeds of an anisotropic, non-linear, torsionally stiff rotor with many degrees of freedom, Journal of Sound and Vibration. 180(1995) 369-386. 